\title{
Directional control of a processive molecular hopper
}

\author{
Authors: Yujia Qing, Sandra A. Ionescu, Gökçe Su Pulcu, and Hagan Bayley*
}

\author{
Affiliations: \\ Department of Chemistry, University of Oxford, 12 Mansfield Road, Oxford, OX1 3TA \\ *Correspondence to: hagan.bayley@chem.ox.ac.uk
}

\begin{abstract}
Intrigued by technological potential, scientists have long attempted to control molecular motion. We monitor the individual 0.7-nm steps of a single molecular hopper as it moves in an electric field along a track in a nanopore controlled by a chemical ratchet. The hopper demonstrates characteristics desired in a moving molecule: defined start- and end-points, processivity, fuel autonomy, directional motion and external control. The hopper is readily functionalized to carry cargos. For example, DNA can be ratcheted along the track in either direction, a prerequisite for nanopore sequencing.
\end{abstract}

One Sentence Summary: A single cargo molecule can be driven back and forth on a track within a protein nanopore.

\section{Main Text:}

Processivity lies at the heart of biological machines. A replicative DNA polymerase can incorporate thousands of nucleotides before dissociating from its template (1). Molecular motors, such as kinesin and dynein, travel directionally along microtubules over hundreds of steps without detaching from the track (2-4). For years, scientists have been trying to build moving molecules that resemble their biomolecular counterparts but use simpler components (5). The ultimate goals are to achieve true processivity, which can be defined as directional motion without leaving a track and the performance of useful work such as the transport of a cargo. Ideally, a synthetic system should exhibit the reversibility of stepping seen in various biological systems $(6,7)$ to enable the direction of motion to be switched through external control.

We report the design of a one-legged molecular hopper that is ratcheted by dynamic covalent chemistry along a protein track (Fig. 1A) with robust processivity (Table S1). Further, the direction in which the hopper moves is subject to external control by an electrical potential. The track is built inside a protein nanopore, $\alpha$-hemolysin $(\alpha \mathrm{HL})$, and consists of a series of cysteine footholds facing the lumen of the transmembrane $\beta$ barrel (Fig. 1B). The cysteines are evenly spaced along a $\beta$ strand with an average inter-foothold distance of $6.8 \AA(\mathrm{C} \alpha-\mathrm{C} \alpha)$ and an average vertical spacing of $5.6 \AA$. The hopper moves in the direction in which the DNA cargo has been oriented by an applied potential, by employing consecutive thiol-disulfide interchange reactions. To execute the $S_{\mathrm{N}} 2$ reaction, the three participating sulfur atoms must align in a near linear configuration (8-10). Under the applied potential, the DNA inside the barrel is pulled in the electric field with a force of $\sim 10 \mathrm{pN}$ (see $\mathrm{SM}$ ). The force sets the overall direction of motion by flipping the DNA (see below) and helps orient the disulfide for cleavage by the neighboring downstream cysteine thiolate, which moves the hopper one step forwards, although other forces can contribute to the forward motion (Fig. 1C). Back-stepping is disfavored and over-stepping is impossible. Release of the hopper from the linear track was not observed, presumably because the track is too rigid to accommodate the resultant disulfide bridge between adjacent footholds on the same $\beta$ strand. In short, each step is chemically directional as the hopper's 'foot' is positioned to favor the forward reaction. Further, the motion is autonomous, requiring no chemical fuel. 
Under $+150 \mathrm{mV}$, the hopper was delivered to the track from the cis compartment as a hoppercarrier conjugate (Fig. 1D) capped with a single traptavidin, which was arrested at the pore entrance (Fig. 2A). The disulfide in the construct reacted strictly regioselectively with Cys-115 releasing the carrier and placing the hopper-DNA cargo on the starting foothold (Fig. 2A, S1). The location of the hopper was ascertained from the residual current passing through the nanopore, which reflected the length of the DNA located within the $\beta$ barrel when the hopper was at a particular foothold (Fig. S1). By monitoring current changes, we followed the stepwise hopping motion at the single-molecule level in real-time.

The voltage-controlled hopping motion was directional and processive. On a track containing five cysteine footholds $(113,115,117,119,121)$, a hopper carrying an oligoadenosine 40-mer (A40, hopper 1) moved cis to trans under $+150 \mathrm{mV}$, and trans to cis under $-150 \mathrm{mV}$ (Fig. 2B, S2). When the hopper reached a terminal foothold, the sign of the applied potential was reversed in order to reorient (flip) the DNA cargo, and hence the hopper. Alternation between positive and negative potentials repeatedly drove the hopper towards the trans or the cis end of the track. For the DNA to experience a force, at least one negatively-charged phosphodiester bond must lie within the electric field, which drops along the length of the pore's $\beta$ barrel (11). Therefore, in the present nanopore construct, the length of the track is limited and voltage-controlled hopping was demonstrated with up to six footholds (Fig. S3). The hopping direction could be changed by reversing the applied potential at any foothold, showcasing the complete control over directionality and the ability to move a hopper back to the initial foothold after an outing.

In the present system, the applied potential provides an external energy source to produce directional motion (Fig. 2C, see SM). Limited by bilayer stability, the longest records of processive hopping were documented with hopper 1, which completed 249 forward steps in 93 min on a fivecysteine track (113-121) with a mean dwell-time of $\sim 22 \mathrm{~s}$ per foothold (Fig. S2). Dissociation of the hopper from the track was never seen ( $n>30$ outings on different tracks), which implies that substantial improvements on step numbers would be achieved if the stability of the bilayer were improved. In comparison, previous synthetic small-molecule walkers moved directionally for less than 10 steps $(5,12)$. Wild-type kinesins typically exhibit a mean step number of $75-175$ before dissociation $(2,3)$.

The hopping rates for each of the four steps on the five-cysteine track were derived for both the cis-to-trans and trans-to-cis directions at $\mathrm{pH} 8.5$ and displayed differences of less than 40-fold (0.0081 to $0.30 \mathrm{~s}^{-1}$, Table S2-4). Because a thiolate is the reactive nucleophile in disulfide interchanges, the rate differences might arise from variations in the $\mathrm{p} K_{\mathrm{a}}$ values of the foothold thiols, which will be affected by neighboring residues. Previously, an arsenic(III) walker showed an up to a 50-fold difference in attachment rates with the footholds on the same five-cysteine track (113-121) at $\mathrm{pH} 8.0$ (12). In the future, tracks of thiols might be engineered with optimized interfoothold distances and enhanced chemical reactivity to speed up the hopping process. Alternatively, the properties of the reactive sulfur atom in the hopper might be manipulated by flanking functional groups. With both the two-cysteine and the three-cysteine tracks, the influence of voltage on hopping was examined at $\pm 100 \mathrm{mV}, \pm 150 \mathrm{mV}$, and $\pm 180 \mathrm{mV}$ (Table S5-6). The rates showed weak non-exponential voltage-dependences suggesting that the applied potential might not be the only source of propulsion (see SM). However, an electrical potential is essential 1) to flip the DNA over a large barrier to set the direction of motion; 2) to aid in the orientation of the three participating sulfur atoms to favor "forward" reactions over back-steps. With respect to the latter, the estimated "effective concentrations" of participating downstream thiols are not 
especially high (see SM) (13) and indeed need not be to produce overall forward motion (see below).

Although disfavored, back-stepping was occasionally detected and attributed to conformational lability of the hopper within the nanopore even under an applied potential. During a recording with hopper 1 on a five-cysteine track (113-121), there were 33 backward steps on the non-terminating footholds out of 282 steps in total (12\%). Of the 33 backward steps, 29 occurred from 115 to 117 at $-150 \mathrm{mV}$ (Table S3). Despite the large forward equilibrium constant for 117 to $115\left(K=k_{117-115} / k_{115-117}=22\right), 115-117$ back-stepping is observed, because of the comparatively slow forward movement to the next foothold 113 (at $-150 \mathrm{mV}, k_{115-117}=0.0094 \mathrm{~s}^{-1} ; k_{115-113}=0.0081$ $\mathrm{s}^{-1}$, Table S2-3). Back-stepping was observed when the hopper was left on a terminal foothold as no forward footholds remained (Fig. 2B). These back-steps were quickly reversed by the hopper, which preferentially resided at the final station $\left(K=k_{119-121} / k_{121-119}=5.2\right.$, Table S4). The overall motion of the hopper is governed by the product of the $K$ values for each step. A modest value ( $K$ $>1$ ) at each step produces a considerable overall tendency towards forward movement.

Each subunit of the $\alpha \mathrm{HL}$ pore offers two antiparallel $\beta$ strands to the transmembrane $\beta$ barrel with an inter-strand distance of $\sim 5 \AA(\mathrm{C} \alpha-\mathrm{C} \alpha)$. Given that the formation of cross-strand disulfides has been reported (14), we reasoned that the addition of a cysteine on an adjacent strand would compel hopper release from the track at a designated foothold. Indeed, with an L-shaped track consisting of cysteines at positions $115,117,119$, and 139, the hopper attached to the track at foothold 115 by regioselective disulfide formation and dissociated from the track when it reached foothold 119. The release was initiated by Cys-139, through thiol-disulfide interchange to form a cross-strand disulfide bridge, which blocked the access of subsequent hoppers to foothold 119 (Fig. 2D). The preference for hopper release versus hopper transfer to the adjacent strand is attributed to the failure of the three participating sulfur atoms to form the co-linear alignment necessary for transfer. In the future, the engineering of footholds on a surface will allow the construction of more complex hopping pathways where hoppers are transferred to new tracks at designed junctions and cargos are released at predesignated depots.

The ability to translocate a stretched DNA cargo while maintaining a covalent bond with the nanopore suggests a method for the chemical ratcheting of a nucleic acid during nanopore sequencing (15), which was explored in a proof-of-concept experiment. To provide a marker, two adjacent abasic residues $\left(1^{\prime}, 2^{\prime}\right.$-dideoxyribose, dS) (16) were incorporated into the cargo oligos carried by hoppers 2 and 3, and current patterns were recorded during four-step hopping between Cys-113 and Cys-121 (Fig. 3A). By comparison with hopper 1, hoppers 2 and 3 showed different patterns of current modulation (Fig. 3BC, S4, Table S7). The conductance patterns generated by the four-step hopping motion could be repeated with different molecules of hopper 2 and hopper 3 ( $n=3$ for each hopper), establishing the patterns as clear identifiers of each cargo sequence. The residual currents (Ires\%, the remaining current as a percentage of the open pore current) for the three hoppers residing at each foothold were plotted for comparison (Fig. 3D). Hopper 1 and hopper 2 gave almost identical current blockades at each of footholds 115 and 113 under $-150 \mathrm{mV}$, implying that the dSdS sequence had been transported well out of the sensing region by hopper 2 . Moreover, hopper 2 and hopper 3 have a single nucleotide offset in the dSdS positions and we observed a one-step offset between hopper 2 and hopper 3 in $\Delta \operatorname{Ires} \%$, the difference in Ires $\%$ between two successive steps (Fig. 3D; the vertical step size, $5.6 \AA$, is similar to the internucleotide distance in stretched single-stranded DNA, $6.9 \AA$ (17)). These observations demonstrate that the hopper system reported here has the potential to discriminate bases for sequencing purposes (16). An advantage of a processive hopper, which might improve sequencing accuracy, 
is the ability to reverse the chemical ratcheting process and thereby obtain many-fold coverage of an individual DNA strand. Of course, the present system is limited by its short track. While longer $\beta$-barrel pores exist $(18,19)$, a viable sequencing process will require protracted ratcheting of numerous DNA strands in parallel, perhaps by using footholds on an extended crystalline surface or internal thiophosphate feet to transport long replica strands over relatively short tracks.

\section{References:}

1. W. A. Breyer, B. W. Matthews, A structural basis for processivity. Protein Sci. 10, 16991711 (2001).

2. R. D. Vale et al., Direct observation of single kinesin molecules moving along microtubules. Nature 380, 451-453 (1996).

3. R. B. Case, D. W. Pierce, N. Hom-Booher, C. L. Hart, R. D. Vale, The directional preference of kinesin motors is specified by an element outside of the motor catalytic domain. Cell 90, 959-66 (1997).

4. A. Gennerich, R. D. Vale, Walking the walk: how kinesin and dynein coordinate their steps. Curr. Opin. Cell Biol. 21, 59-67 (2009).

5. S. Erbas-Cakmak, D. A. Leigh, C. T. McTernan, A. L. Nussbaumer, Artificial molecular machines. Chem. Rev. 115, 10081-10206 (2015).

6. W. J. Walter, M. P. Koonce, B. Brenner, W. Steffen, Two independent switches regulate cytoplasmic dynein's processivity and directionality. Proc. Natl. Acad. Sci. U. S. A. 109, 5289-93 (2012).

7. R. A. Cross, A. McAinsh, Prime movers: the mechanochemistry of mitotic kinesins. Nat. Rev. Mol. Cell Biol. 15, 257-71 (2014).

8. R. D. Bach, O. Dmitrenko, C. Thorpe, Mechanism of thiolate-disulfide interchange reactions in biochemistry. J. Org. Chem. 73, 12-21 (2008).

9. P. A. Fernandes, M. J. Ramos, Theoretical insights into the mechanism for thiol/disulfide exchange. Chem. - A Eur. J. 10, 257-266 (2004).

10. A. P. Wiita et al., Probing the chemistry of thioredoxin catalysis with force. Nature 450, 124-127 (2007).

11. S. Howorka, H. Bayley, Probing distance and electrical potential within a protein pore with tethered DNA. Biophys. J. 83, 3202-3210 (2002).

12. G. S. Pulcu, E. Mikhailova, L.-S. Choi, H. Bayley, Continuous observation of the stochastic motion of an individual small-molecule walker. Nat. Nanotechnol. 10, 76-83 (2015).

13. C. A. Hunter, H. L. Anderson, What is cooperativity? Angew. Chemie Int. Ed. 48, 74887499 (2009).

14. J. Venkatraman, G. A. Nagana Gowda, P. Balaram, Design and construction of an open multistranded $\beta$-sheet polypeptide stabilized by a disulfide bridge. J. Am. Chem. Soc. 124, 4987-94 (2002).

15. H. Bayley, Nanopore sequencing: from imagination to reality. Clin. Chem. 61, 25-31 (2015).

16. B. Gyarfas et al., Mapping the position of DNA polymerase-bound DNA templates in a nanopore at $5 \AA$ resolution. ACS Nano 3, 1457-66 (2009).

17. A. Bosco, J. Camunas-Soler, F. Ritort, Elastic properties and secondary structure formation of single-stranded DNA at monovalent and divalent salt conditions. Nucleic Acids Res. 42 , 2064-2074 (2014).

18. I. Iacovache et al., Cryo-EM structure of aerolysin variants reveals a novel protein fold and the pore-formation process. Nat. Commun. 7, 12062 (2016). 
19. J. Jiang, B. L. Pentelute, R. J. Collier, Z. H. Zhou, Atomic structure of anthrax protective antigen pore elucidates toxin translocation. Nature 521, 545-549 (2015).

20. M. von Delius, E. M. Geertsema, D. A. Leigh, A synthetic small molecule that can walk down a track. Nat. Chem. 2, 96-101 (2010).

21. M. R. Wilson et al., An autonomous chemically fuelled small-molecule motor. Nature 534, 235-40 (2016).

22. S. Erbas-Cakmak et al., Rotary and linear molecular motors driven by pulses of a chemical fuel. Science 358, 340-343 (2017).

23. A. G. Campaña et al., A small molecule that walks non-directionally along a track without external intervention. Angew. Chemie Int. Ed. 51, 5480-5483 (2012).

24. A. G. Campaña, D. A. Leigh, U. Lewandowska, One-dimensional random walk of a synthetic small molecule toward a thermodynamic sink. J. Am. Chem. Soc. 135, 8639-45 (2013).

25. M. J. Barrell, A. G. Campaña, M. von Delius, E. M. Geertsema, D. A. Leigh, Light-driven transport of a molecular walker in either direction along a molecular track. Angew. Chemie Int. Ed. 50, 285-290 (2011).

26. J. E. Beves et al., Toward metal complexes that can directionally walk along tracks: controlled stepping of a molecular biped with a palladium(II) foot. J. Am. Chem. Soc. 136, 2094-100 (2014).

27. Y. He, D. R. Liu, Autonomous multistep organic synthesis in a single isothermal solution mediated by a DNA walker. Nat. Nanotechnol. 5, 778-782 (2010).

28. Y. Tian, Y. He, Y. Chen, P. Yin, C. Mao, A DNAzyme that walks processively and autonomously along a one-dimensional track. Angew. Chemie Int. Ed. 44, 4355-8 (2005).

29. M. You et al., An autonomous and controllable light-driven DNA walking device. Angew. Chemie Int. Ed. 51, 2457-2460 (2012).

30. T.-G. Cha et al., A synthetic DNA motor that transports nanoparticles along carbon nanotubes. Nat. Nanotechnol. 9, 39-43 (2013).

31. K. Yehl et al., High-speed DNA-based rolling motors powered by RNase H. Nat. Nanotechnol. 11, 184-190 (2015).

32. S. F. J. Wickham et al., Direct observation of stepwise movement of a synthetic molecular transporter. Nat. Nanotechnol. 6, 166-169 (2011).

33. H. Gu, J. Chao, S.-J. Xiao, N. C. Seeman, A proximity-based programmable DNA nanoscale assembly line. Nature 465, 202-5 (2010).

34. J.-S. Shin, N. A. Pierce, A synthetic DNA walker for molecular transport. J. Am. Chem. Soc. 126, 10834-5 (2004).

35. B. Ibarra et al., Proofreading dynamics of a processive DNA polymerase. EMBO J. 28, 2794-802 (2009).

36. A. B. C. Deutman et al., Mechanism of threading a polymer through a macrocyclic ring. Science 322, 1668-1671 (2008).

37. N. Koumura, R. W. J. Zijlstra, R. A. van Delden, N. Harada, B. L. Feringa, Light-driven monodirectional molecular rotor. Nature 401, 152-155 (1999).

38. S. Kassem et al., Stereodivergent synthesis with a programmable molecular machine. Nature 549, 374-378 (2017).

39. C. Cheng et al., An artificial molecular pump. Nat. Nanotechnol. 10, 547-553 (2015).

40. J. Nakane, M. Wiggin, A. Marziali, A nanosensor for transmembrane capture and identification of single nucleic Acid molecules. Biophys. J. 87, 615-21 (2004). 
41. Y. Wang, K. Tian, L. L. Hunter, B. Ritzo, L.-Q. Gu, Probing molecular pathways for DNA orientational trapping, unzipping and translocation in nanopores by using a tunable overhang sensor. Nanoscale 6, 11372-11379 (2014).

42. R. P. Szajewski, G. M. Whitesides, Rate constants and equilibrium constants for thioldisulfide interchange reactions involving oxidized glutathione. J. Am. Chem. Soc. 102, 2011-2026 (1980).

43. D. M. Rothwarf, H. A. Scheraga, Equilibrium and kinetic constants for the thiol-disulfide interchange reaction between glutathione and dithiothreitol. Proc. Natl. Acad. Sci. U. S. A. 89, 7944-8 (1992).

44. D. A. Keire, E. Strauss, W. Guo, B. Noszal, D. L. Rabenstein, Kinetics and equilibria of thiol/disulfide interchange reactions of selected biological thiols and related molecules with oxidized glutathione. J. Org. Chem. 57, 123-127 (1992).

45. A. F. Hammerstein, S.-H. Shin, H. Bayley, Single-molecule kinetics of two-step divalent cation chelation. Angew. Chemie Int. Ed. 49, 5085-5090 (2010).

46. V. M. Krishnamurthy, V. Semetey, P. J. Bracher, N. Shen, G. M. Whitesides, Dependence of effective molarity on linker length for an intramolecular protein-ligand system. J. Am. Chem. Soc. 129, 1312-1320 (2007).

47. J. Lee et al., Semisynthetic nanoreactor for reversible single-molecule covalent chemistry. ACS Nano 10, 8843-8850 (2016).

48. G. Miles, H. Bayley, S. Cheley, Properties of bacillus cereus hemolysin II: a heptameric transmembrane pore. Protein Sci. 11, 1813-1824 (2009).

49. M. Montal, P. Mueller, Formation of bimolecular membranes from lipid monolayers and a study of their electrical properties. Proc. Natl. Acad. Sci. U. S. A. 69, 3561-3566 (1972).

50. C. Nicolai, F. Sachs, Solving ion channel kinetics with the QuB software. Biophys. Rev. Lett. 8, 191-211 (2013).

51. F. Qin, A. Auerbach, F. Sachs, Estimating single-channel kinetic parameters from idealized patch-clamp data containing missed events. Biophys. J. 70, 264-280 (1996).

Acknowledgments: We thank Dr Hiroko Tamagaki and Dr Joongoo Lee for their help with peptide design and synthesis. We thank Dr Sejeong Lee for her help with data analysis using QuB. We also thank Lapatrada Taemaitree for her help with LC-MS characterization. We thank Professor Mark Howarth for providing a sample of traptavidin for preliminary tests. Funding: This research was supported by a European Research Council Advanced Grant. Y.Q. was supported by the China Scholarship Council-University of Oxford Scholarship. S.A.I. is funded by the Amelia Jackson Studentship, Exeter College, Oxford; Author contributions: Y.Q. and H.B. designed the study, Y.Q. performed the experiments, S.A.I. repeated the single-channel recordings, G.S.P. contributed to the protein preparation, Y.Q., S.A.I. and H.B. wrote the manuscript. Competing interests: H.B. is the founder of, a consultant for, and a share-holder of Oxford Nanopore Technologies, a company engaged in the development of nanopore sensing and sequencing technologies. A patent has been filed covering the molecular hopper and applications thereof. Data and materials availability: All data are available in the main text or the supplementary materials.

\section{Supplementary Materials:}

Materials and Methods 
Figures S1-S12

Tables S1-S7

Movies S1-S2

References 20-51 


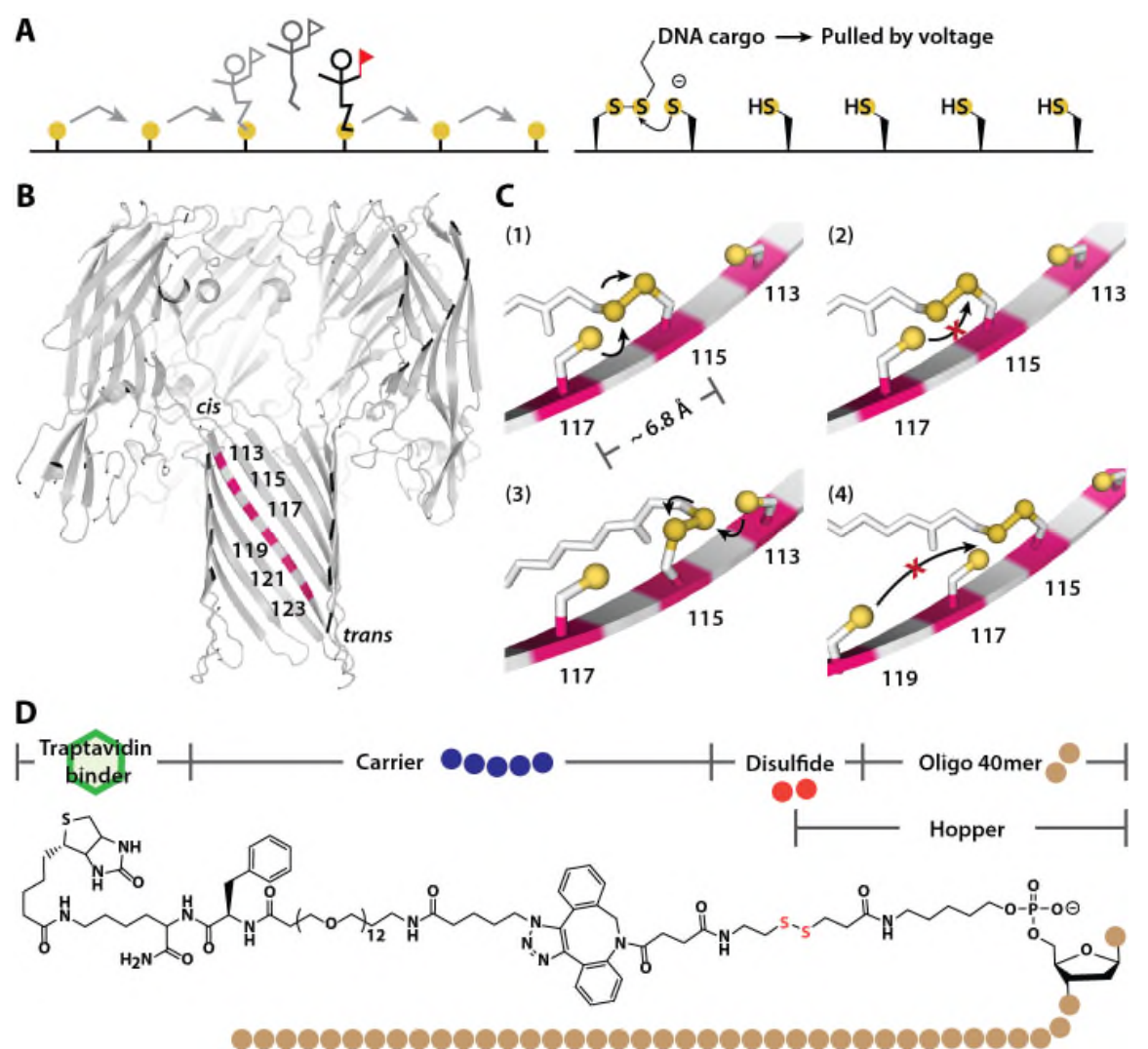

Fig. 1. A molecular hopper on a protein track. (A) A hopper carrying a cargo (red flag) moves along a track, by employing consecutive thiol-disulfide interchange reactions. The overall direction is set by the applied voltage. (B) A six-foothold track comprising odd-numbered cysteine residues on a $\beta$ strand inside the $\alpha \mathrm{HL}$ protein nanopore. (C) The applied potential exerts a force on the DNA cargo, which helps align the three sulfur atoms (yellow) participating in the interchange. The colinear geometry promotes hopping (1) (Supplementary Movie 1), but not the formation of an intra-strand disulfide, which would release the hopper from the track (2). Occasionally, back-stepping is observed (3). Over-stepping (4) does not occur. (D) The hopper enters the nanopore as a carrier-hopper disulfide conjugate. 
A

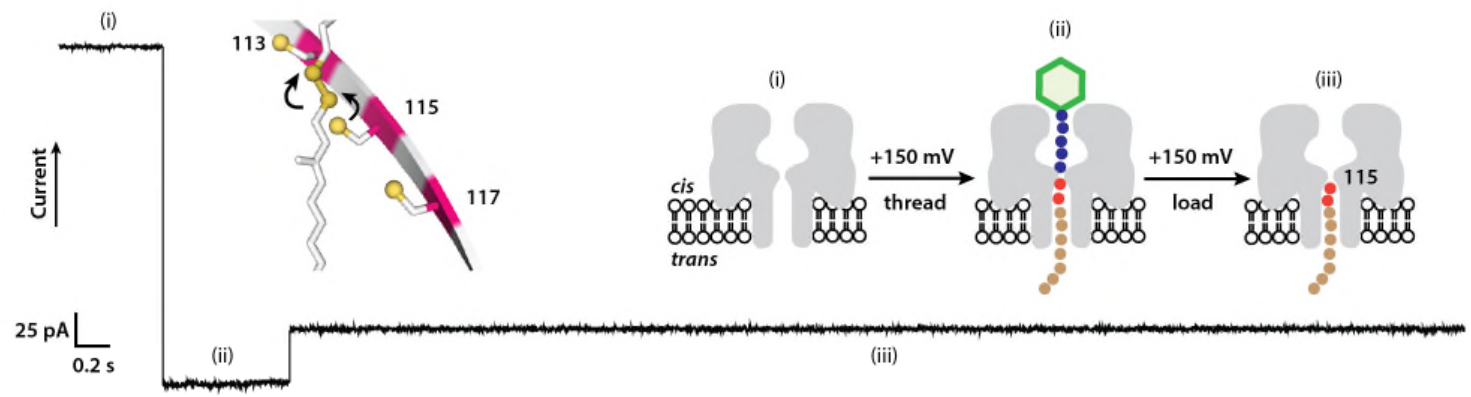

B

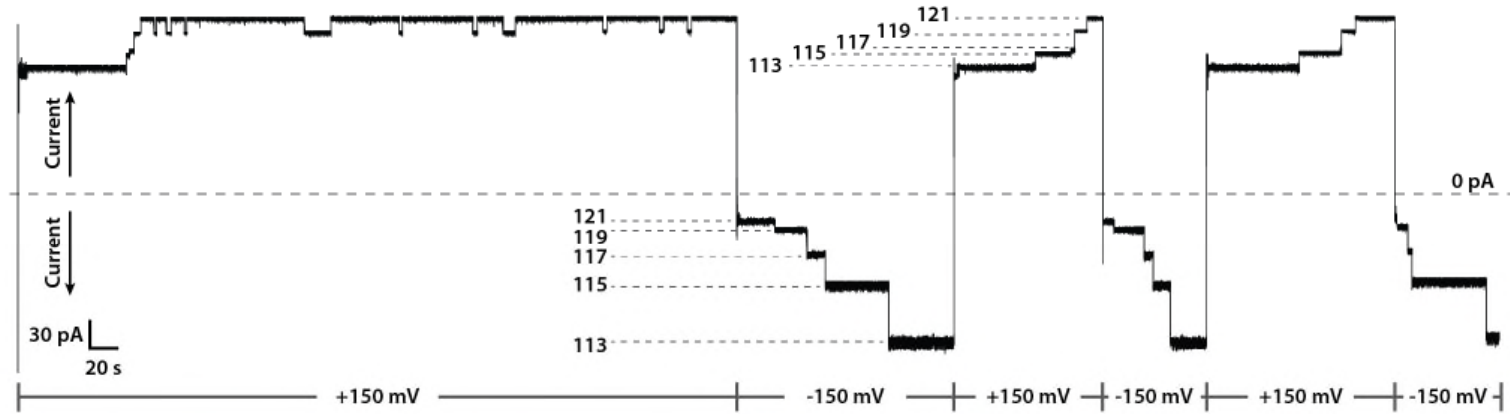

C

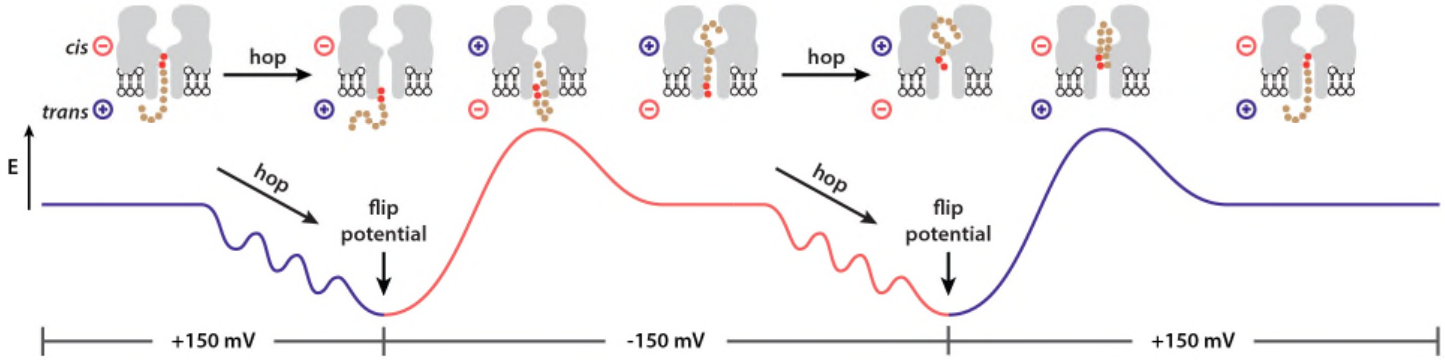

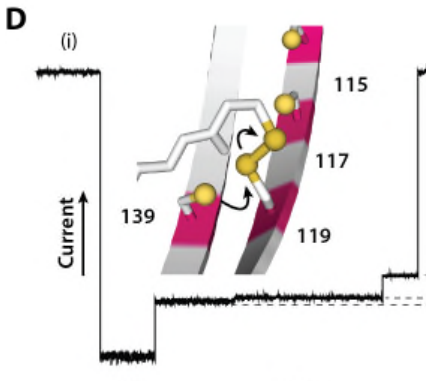

(ii)

(iii)
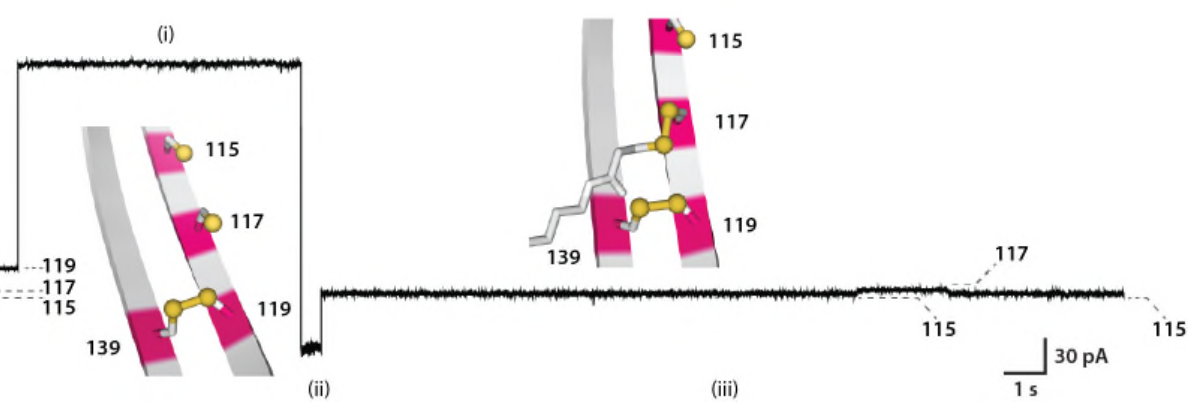

Fig. 2. Monitoring individual hopper steps. (A) Under $+150 \mathrm{mV}$, a hopper-carrier conjugate capped with traptavidin was pulled from the cis compartment into an $\alpha \mathrm{HL}$ nanopore containing cysteines at positions $113,115,117,119$, and 121 in one of the seven subunits. The resultant blockade reduced the ionic current from (i) to (ii). Reaction of the disulfide in the hopper-carrier with Cys-115 covalently attached the hopper to the track, and the ionic current increased to (iii). (B) With a five-cysteine track, four hopping steps were observed at $\pm 150 \mathrm{mV}$. Every forward step moved part of the DNA cargo outside the $\beta$ barrel, producing an increase in conductance (Supplementary Movie 2). Alternation of the applied potential drove the hopper repeatedly up and down the track. (C) A hypothetical free energy diagram (not to scale) of the controlled hopping 
motion. (D) On an L-shaped track consisting of cysteines at positions 115, 117, 119, and 139, the hopper moved along the track from Cys-115 to Cys-119, where it was released by the side chain of Cys-139. Subsequently, a second hopper became loaded at Cys-115, but its motion was arrested at Cys-117 because Cys-119 was now engaged in an inter-strand disulfide bond. Back-stepping to Cys-115 was also seen. Conditions: $2 \mathrm{M} \mathrm{KCl}, 20 \mathrm{mM}$ HEPBS, $20 \mu \mathrm{M}$ EDTA, pH 8.5, $20 \pm 1{ }^{\circ} \mathrm{C}$. 

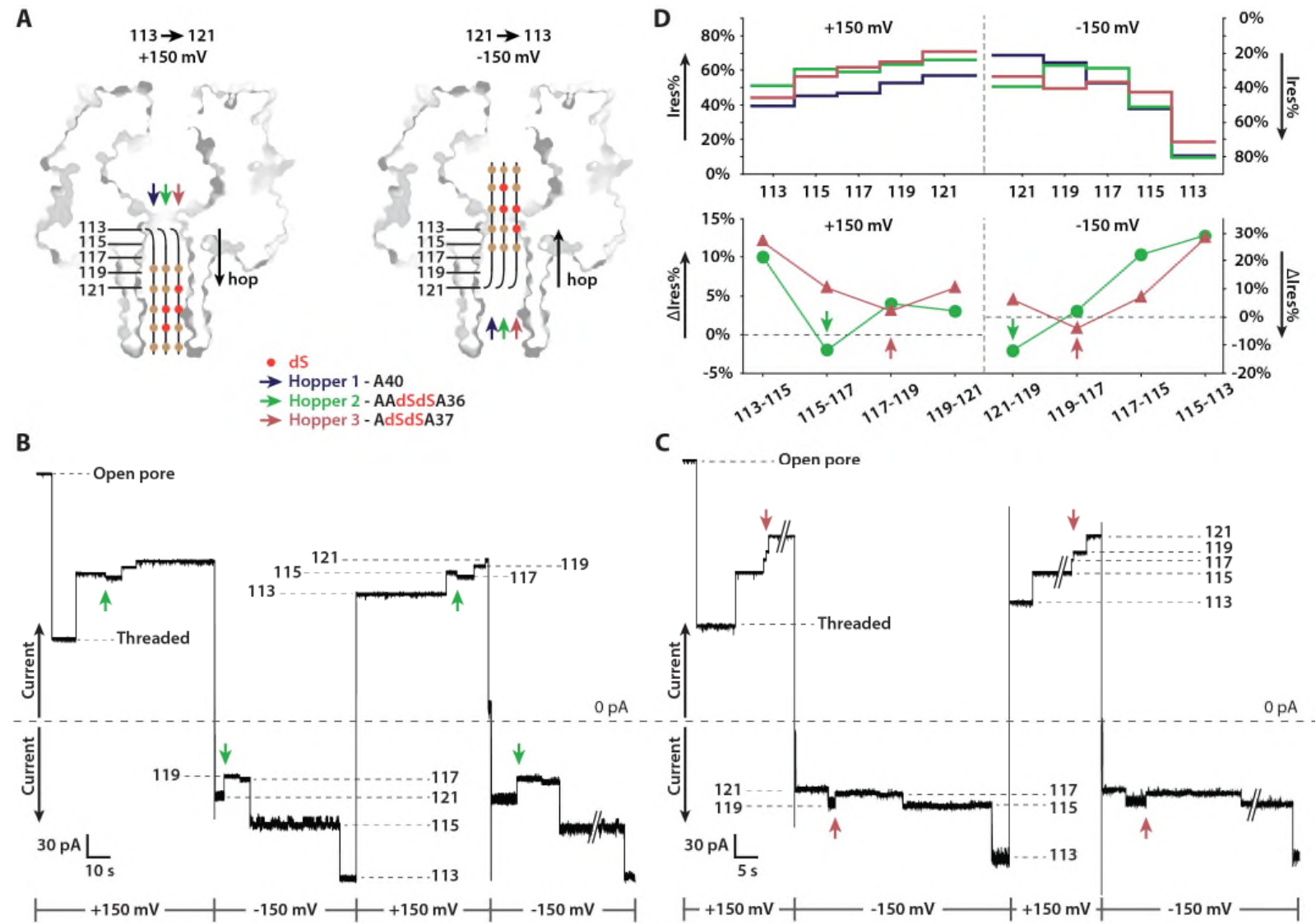

Fig. 3. Discrimination of different DNA cargos. (A) Two sequential abasic nucleotides (dSdS) were substituted at positions 3 and 4 (hopper 2) or at positions 2 and 3 (hopper 3). The numbers of nucleotides (brown circles, dA; red circles dS) placed inside the $\beta$ barrel are based on PyMOL modelling. (B) With a five-cysteine track, four-step hopping was observed with hopper 2 at \pm 150 $\mathrm{mV}$. The current decreases for hops from 115 to 117 and from 121 to 119 are marked (green arrows). (C) Four-step hopping with hopper 3 at $\pm 150 \mathrm{mV}$. The current transitions for hops from 117 to 119 and from 119 to 117 are marked (red arrows). (D) Top: Overlaid current traces of hoppers 1,2, and 3 with step durations normalized. The current levels are given as the residual current with respect to the open pore level (Ires\%). Bottom: Step sizes of hoppers 2 and 3 plotted as $\Delta$ Ires\%. Minima in the plots showing the single nucleotide offset are marked. Conditions: $2 \mathrm{M}$

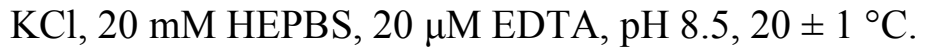

